\title{
UNIVERSITY OF KIEL RADIOCARBON MEASUREMENTS IV
}

\author{
H. WILLKOMM and H. ERLENKEUSER \\ $\mathrm{C}^{14}$ Laboratory, University of Kiel, Germany
}

The following dates are samples measured since publication of Kiel III (Radiocarbon, v. 10, p. 328-332). Age calculations are based on 95\% of the activity of NBS oxalic-acid standard as modern value of A.D. 1950. Results are calculated with Libby half-life and reported in yr before 1950. Error corresponds to $1 \sigma$ variation of sample net counting rate as well as modern standard and background, but does not include the uncertainty in $\mathrm{C}^{14}$ half-life and in secular $\mathrm{C}^{14}$ variations. Dates are not corrected for isotopic fractionation.

Most of the measurements have been obtained with a 4.5-L CO. counter; smaller samples were measured in a $3-\mathrm{L}$ counter, and about $20 \%$ of the samples were measured with both counters.

In 1968, we constructed two additional $\mathrm{CO}_{2}$ counters. One is a proportional counter with a separate ring counter for shielding cosmic radiation. The other is an Oeschger-type counter (Hänsel, 1968). Both counters are made of commercially available copper. Insulating parts are built of teflon heated in vacuum for several days at $180^{\circ} \mathrm{C}$. Nevertheless, the Oeschger counter still degasses, but it is possible to measure a sample up to one week in this counter. For working values, see Table 1.

TABIE 1

$\mathrm{CO}_{2}$ counters of the $\mathrm{C}^{14}$ Laboratory, Kiel

\begin{tabular}{|c|c|c|c|c|}
\hline Counter & $\mathrm{a}$ & $\mathrm{b}$ & $\mathrm{c}$ & d \\
\hline Type & $\begin{array}{l}\text { Prop. counter } \\
\quad 36 \\
\text { separate GM- } \\
\text { for screening }\end{array}$ & $\begin{array}{l}\text { with } \\
28 \\
\text { counters }\end{array}$ & $\begin{array}{l}\text { Prop. counter } \\
\text { with a ring } \\
\text { counter for } \\
\text { screening }\end{array}$ & $\begin{array}{l}\text { Oeschger } \\
\text { counter }\end{array}$ \\
\hline $\begin{array}{l}\text { Internal } \\
\text { shielding }\end{array}$ & $\begin{array}{l}\text { Quartz tube } \\
\text { as cathode }\end{array}$ & - & - & $\begin{array}{l}75 \mu \text { Hostaphan } \\
\text { foil with vapor- } \\
\text { ized Aluminum on } \\
\text { both sides to } \\
\text { separate inner } \\
\text { and outer part }\end{array}$ \\
\hline $\begin{array}{l}\text { Effective } \\
\text { volume }\end{array}$ & $4.5 \mathrm{~L}$ & $3.0 \mathrm{~L}$ & $3.0 \mathrm{~L}$ & $\begin{array}{l}4.2 \mathrm{~L} \\
(\text { total } 12.6 \mathrm{~L})\end{array}$ \\
\hline Pressure & 500 torr & 600 torr & 1000 torr & 500 torr \\
\hline $\begin{array}{l}\text { Recent value } \\
(95 \% \text { of oxalic } \\
\text { acid, calculated } \\
\text { for A.D. } 1950) \\
\text { Background }\end{array}$ & 21.28 & 13.94 & 22.54 & $16.51 \mathrm{cpm}$ \\
\hline
\end{tabular}


Counting rates of the last dating equipment (guard counter, central counter, coincidences of inner and outer part, and central pulses, which do not coincide with a guard pulse) are punched in a tape every 100th minute. When measurement is finished the tape can be given to computer without any corrections and can be evaluated by an ALGOL program. We intend to give the counting rates of all 4 dating equipments on a single tape perforator (for details, see Hänsel, 1968).

\section{Sehestedt series}

$$
\text { I. GEOLOGIC SAMPLES }
$$

Peat samples from bog (53 $26^{\prime} \mathrm{N}$ Lat, $8^{\circ} 18^{\prime} \mathrm{E}$ Long) at Jadebusen (German shore of North Sea). Coll. for investigation of settlement in NW Germany by R. Wiermann, who also made pollen analysis, Bot. Inst., Univ. Münster. Subm. 1965 by F. Overbeck, Bot. Inst., Univ. Kiel. Comment (R.W.): samples KI-162 and KI-161 show beginning, KI-160 shows end of interruption in settlement. Pollen of KI-159 to KI-155 represent intensive medieval agriculture, which stops in KI-155. Youngest interruption is represented by KI-154 and KI-153.

General Comment: substituting Libby-age by real astronomical age (for calculation, see Willkomm, 1968), ages of samples correspond within statistical error with growth rate of $150 \mathrm{~cm}$ per millennium.

\begin{tabular}{|c|c|c|c|}
\hline KI-153. & Seh-I,1 & 4 to $9 \mathrm{~cm}$ depth & A.D. 1600 \\
\hline KI-154. & Seh-I,2 & 9 to $14 \mathrm{~cm}$ depth & $\begin{aligned} & 475 \\
& \text { A.D. } 1475\end{aligned}$ \\
\hline KI-155. & Seh-I,3 & 50 to $55 \mathrm{~cm}$ depth & $\begin{array}{r}1060 \pm 85 \\
\text { A.D. } 890\end{array}$ \\
\hline KI-156. & Seh-I,4 & 55 to $60 \mathrm{~cm}$ depth & $\begin{aligned} 840 & \pm 45 \\
\text { A.d. } 1110 & \end{aligned}$ \\
\hline KI-157. & Seh-I,5 & 60 to $65 \mathrm{~cm}$ depth & $\begin{aligned} 920 & \pm 35 \\
\text { A.d. } 1030 & \end{aligned}$ \\
\hline KI-158. & Seh-I, 6 & 65 to $70 \mathrm{~cm}$ depth & $\begin{array}{r}900 \pm 55 \\
\text { A.D. } 1050\end{array}$ \\
\hline KI-159. & Seh-I,7 & 70 to $75 \mathrm{~cm}$ depth & $\begin{array}{r}950 \pm 30 \\
\text { A.D. } 1000\end{array}$ \\
\hline KI-160. & Seh-1,8 & 145 to $150 \mathrm{~cm}$ depth & $\begin{array}{r}1305 \\
\text { A.D. } 595\end{array}$ \\
\hline KI-161. & Seh-I,9 & 190 to $195 \mathrm{~cm}$ depth & $\begin{array}{l}1830 \pm 50 \\
\text { A.D. } 120\end{array}$ \\
\hline KI-162. & Seh-I,10 & 195 to $200 \mathrm{~cm}$ depth & $\begin{array}{l}1750 \pm 60 \\
\text { A.D. } 200\end{array}$ \\
\hline KI-163. & h-I, I1 & 220 to 2 & $\begin{array}{l}1930 \pm 50 \\
\text { A.d. } 20\end{array}$ \\
\hline
\end{tabular}

Large amounts of Carpinus pollen. 
KI-164. Seh-I, $12 \quad 235$ to $240 \mathrm{~cm}$ depth

Large amounts of Fagus pollen.

\section{Meerhusener Moor series}

Peat samples from "Meerhusener Moor", bog (53 $32^{\prime} \mathrm{N}$ Lat, $7^{\circ} 30^{\prime}$ E Long, r 2597 740, h 5935 130) near Aurich, NW Germany. Coll. 1967 and subm. 1968 by H. Usinger, Bot. Inst., Univ. Kiel. Comment (H.U.): KI-252 lay directly below artificial sandy way across bog, which later was abandoned and overgrown by bog. KI-251 comes from layer directly above way.

KI-251. $84 \mathrm{~cm}$ depth

KI-252. $151 \mathrm{~cm}$ depth

$$
\begin{aligned}
1830 & \pm 65 \\
\text { A.D. } 120 & \\
2440 & \pm 40 \\
490 & \text { B.C. }
\end{aligned}
$$

\section{Keitum series}

Peat from Tinnum, Keitum, and Archsum on Sylt I. (54 $54^{\prime} \mathrm{N}$ Lat, $8^{\circ} 23^{\prime}$ E Long), NW Germany. Coll. and subm. 1967 by D. Hoffman, Germany by R. Wiermann, who also made pollen analysis, Bot. Inst., Geol. Inst., Univ. Kiel. Comment (D.H.): a few thousand yr ago, lower part of E Sylt I. was covered by bogs, which now yield peat layer up to $180 \mathrm{~cm}$ thickness. Due to following transgression by North Sea, this layer was covered by clay (marshy soil). Peat samples were taken from boundary

\begin{tabular}{|c|c|c|}
\hline KI-99. & $\begin{array}{l}\text { Tinnum, r } 3456300, \text { h } 6085620 \text {, } \\
50 \mathrm{~cm}\end{array}$ & $\begin{array}{l}2000 \pm 85 \\
50 \text { B.c. }\end{array}$ \\
\hline KI-202. & n, r 3456340, h 6085070 & $\begin{array}{l} \pm 60 \\
\text { B.c. }\end{array}$ \\
\hline KI-96. & og, r 3456220, h 6084370 & $\begin{aligned} 430 & \pm 45 \\
\text { A.d. } 1520 & -40\end{aligned}$ \\
\hline KI-97. & r 3456500, h 6084090 & $\begin{array}{l}3300 \pm 50 \\
1350 \text { в.C. }\end{array}$ \\
\hline KI-204. & $\begin{array}{l}\text { Tinnum, r } 3456300, \text { h } 6085620 \text {, } \\
30 \mathrm{~cm}\end{array}$ & $\begin{aligned} & 1710 \pm 60 \\
& \text { A.D. } 240\end{aligned}$ \\
\hline KI-95. & r 3460480, h 6084460 & $\begin{array}{l}4620 \pm 50 \\
2670 \text { B.c. }\end{array}$ \\
\hline KI-200. & , 3460510, h 6084430 & $\begin{array}{l}3690 \pm 50 \\
1740 \text { B.C. }\end{array}$ \\
\hline KI-100 & $\begin{array}{l}\text { Keitum, r } 3459100, \text { h } 6083570 \text {, } \\
240 \mathrm{~cm}\end{array}$ & $\begin{array}{l}3820 \pm 60 \\
1870 \text { B.C. }\end{array}$ \\
\hline
\end{tabular}
of peat to clay at different locations to date time of transgression.

For each sample, location, coordinates of Topographische Karte 1116 Morsum and depth are given. 
KI-201. Keitum, r 3459550 , h 6082840 ,

KI.98. Archsum, r 3462 050, h 6081250 , $220 \mathrm{~cm}$

KI-203. Archsum, r 3461 790, h 6080330 , $250 \mathrm{~cm}$

\section{Föhr Island}

Wood and peat beneath surface of mud near shore of Fonhr I. (54 $41^{\prime}$ N Lat, $8^{\circ} 27^{\prime}$ E Long), Germany. Coll. and subm. 1967 by F. Rüth for dating transgression of region by North Sea.

KI-231. Wood of trunk, 2 to $5 \mathrm{~cm}$ under surface of mud

$$
\begin{aligned}
& 4100 \pm 60 \\
& 2150 \text { B.C. }
\end{aligned}
$$

KI-232. Root of tree, 2 to $5 \mathrm{~cm}$ depth

$$
4240 \pm 60
$$

KI-233. Peat, 10 to $15 \mathrm{~cm}$ depth

$$
4230 \pm 75
$$

KI-234. Root of tree, 10 to $15 \mathrm{~cm}$ depth

\section{Kieler Bucht series}

Peat and wood of borings from bottom of Baltic Sea. Samples coll. for dating transgression of Kieler Bucht. KI-207 to KI-215 coll. 1962 to 1966 and subm. 1967 by F. Werner; KI-216 and KI-217 coll. 1966 and subm. 1967 by F. C. Kögler, both Geol. Inst., Univ. Kiel.

KI-207.

$9430 \pm 85$

7480 B.C

Peat, 34 to $43 \mathrm{~cm}$ depth, $31 \mathrm{~m}$ below sea level $(-31 \mathrm{~m})\left(54^{\circ} 41.8^{\prime} \mathrm{N}\right.$ Lat, $10^{\circ} 9.5^{\prime} \mathrm{E}$ Long).

KI-208.

$9780 \pm 100$

Peat, like KI-207, 43 to $52 \mathrm{~cm}$ depth.

KI-209.

$8150 \pm 80$ 6200 в.с.

Peat, 70 to $80 \mathrm{~cm}$ depth, $-19 \mathrm{~m}\left(54^{\circ} 30.2^{\prime}\right.$ N L at, $10^{\circ} 2^{\prime}$ E Long).

\section{KI-210.}

$8250 \pm 130$

Peat, like KI-209, 91 to $102 \mathrm{~cm}$ depth.

KI-211.

Wood, $20 \mathrm{~cm}$ depth, $-19 \mathrm{~m}$ (54ㅇ $35.9^{\prime} \mathrm{N}$ Lat, $10^{\circ} 20.6^{\prime} \mathrm{E}$ Long).

\section{KI-214.}

Peat, 17 to $24 \mathrm{~cm}$ depth, $-12.5 \mathrm{~m}\left(54^{\circ} 25.4^{\prime} \mathrm{N}\right.$ Lat, $10^{\circ} 55.9^{\prime} \mathrm{E}$ Long). 
KI-215.

$7300 \pm 100$

Wood, like KI-214, $25 \mathrm{~cm}$ depth.

5350 в.c.

KI-216.

$4840 \pm 110$

2890 B.C.

Peat, 92 to $95 \mathrm{~cm}$ depth, $-2.5 \mathrm{~m}\left(54^{\circ} 21^{\prime} \mathrm{N}\right.$ Lat, $11^{\circ} 4^{\prime} \mathrm{E}$ Long).

KI-217.

$5940 \pm 75$

Peat, like KI-216, 115 to $119 \mathrm{~cm}$ depth.

3990 B.C.

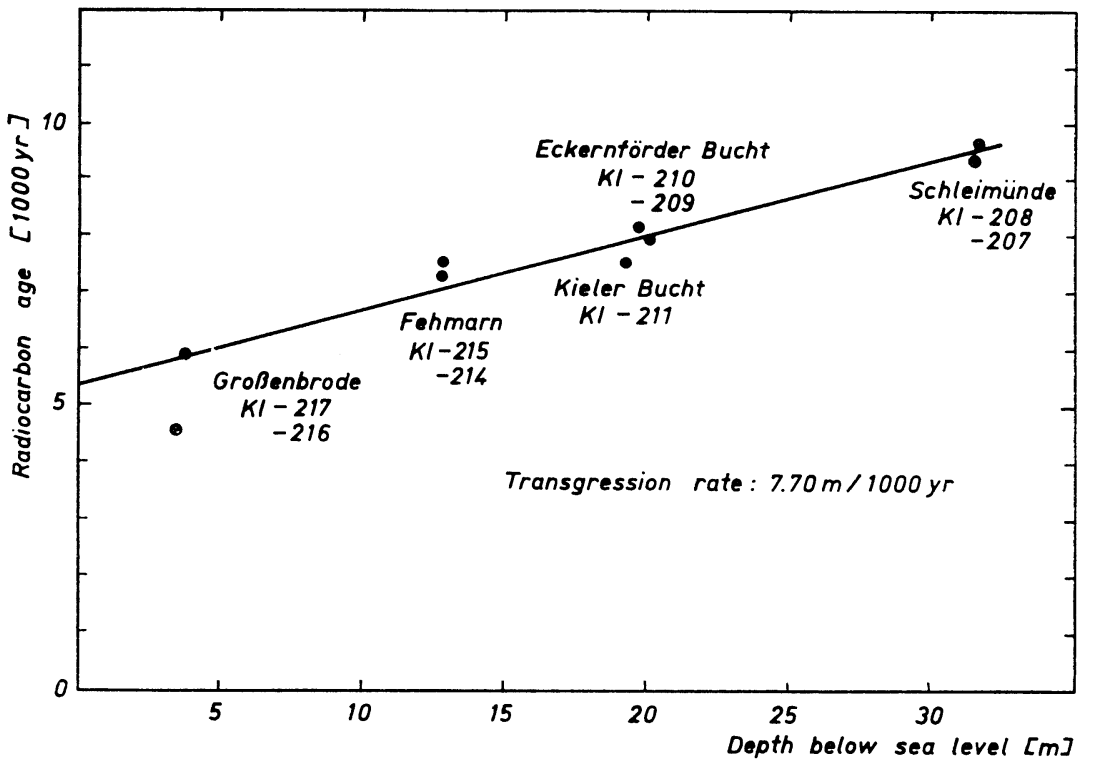

Fig. 1. Transgression of Baltic Sea (Western Part).

\section{Lake Valencia}

Calcareous sediments of Lake Valencia $\left(10^{\circ} 15^{\prime} \mathrm{N}\right.$ Lat, $67^{\circ} 41^{\prime} \mathrm{W}$ Long), Venezuela. Coll. and subm. 1967 and 1968 by F. Gessner, Inst. f. Meereskunde, Univ. Kiel.

General Comment: because modern activity of these samples is unknown, we give only $\delta \mathrm{C}^{14}$ values without age calculation.

\section{KI-93.}

$$
\delta \mathrm{C}^{14}=-3.4 \pm 0.5 \%
$$

Snail shells, $5 \mathrm{~cm}$ depth at $4 \mathrm{~m}$ water depth.

KI-247.

$\delta \mathrm{C}^{14}=-31.8 \pm 0.6 \%$

Sediment of partly inorganic origin, now several $\mathrm{m}$ above lake level.

KI-248.

$\delta \mathrm{C}^{14}=-\mathbf{3 8 . 8} \pm \mathbf{0 . 4 \%}$

Like 247, but from a lower level. 
II. ARCHAEOLOGIC SAMPLES

KI-89. Varna

2260 B.C.

Wood from lake dwellings, now under sea level, at Varna $\left(43^{\circ} 12^{\prime}\right.$ N Lat, $27^{\circ} 55^{\prime}$ E Long), Bulgaria. Coll. 1965 by Mrs. Tonceva, subm. by F. R. Averdieck and R. Kenk, Inst. f. Ur- und Frühgeschichte, Univ. Kiel.

KI-91. Hüsby

Wood of coffin of Bronze age, found at Hüsby (54 $30^{\prime} \mathrm{N}$ Lat, $9^{\circ} 29^{\prime}$ E Long), Germany. Coll. 1966 and subm. 1967 by K. Kersten, SchleswigHolsteinisches Landesmus., Schleswig, Germany. Tree coffin lay in center of tumulus $200 \mathrm{~cm}$ deep.

\section{KI-221. Hüsby}

$3160 \pm 75$

Moss and twigs covering coffin KI-91. Coll. 1966 and subm. 1967 by F. R. Averdieck.

\section{Westerkappeln series}

Charcoal from 2 different layers within dune near Westerkappeln (r 3419 760, h 5803 580), Germany. Coll. 1966 and subm. 1967 by K. Günther, Landesmus. f. Vor- und Frühgeschichte Münster, Germany. Samples were supposed to represent 2 different cultures of late Palaeolithic or Mesolithic age (Winkelmann, 1959).

\section{KI-205. Westerkappeln $1966 / 2$}

$3610 \pm 45$

Lower cultural layer, $30 \mathrm{~cm}$ under KI-206.

\section{KI-206. Westerkappeln 1966/1}

Comment (K.G.): possibly $\mathrm{C}^{14}$ dates, which are much too young, can be explained by wind blowing, which brought essentially younger charcoal into deeper layers.

\section{Möllenknob series}

Excavations near Archsum (54 $52.7^{\prime} \mathrm{N}$ Lat, $8^{\circ} 22.5^{\prime}$ E Long) on Sylt I., Germany (Radiocarbon, 1968, v. 10, p. 331). Coll. 1967 by R. Kenk; subm. 1968 by G. Kossack and F. R. Averdieck.

KI-244. Möllenknob 245 (2)

$3060 \pm 65$

Cereals, weeds, and small pieces of charcoal from small ditches. Younger Bronze age or older pre-Roman Iron age.

KI-245. Möllenknob 288 (17)

$2055 \pm 60$

Cereals from storage vessel of a burnt house. Archaeol. estimate ca. A.D. 0 . 
Coll. 1967 and subm. 1968 by K. W. Struve, Landesmus. f. Vor- und Frühgeschichte Schleswig, Germany. Comment (K.W.S.): part of discwheel, which lay together with 2 other disc-wheels of Neolithic or Bronzeage type near Alt-Bennebek (54 $23^{\prime} \mathrm{N}$ Lat, $9^{\circ} 24^{\prime} \mathrm{E}$ Long), Germany.

\section{KI-262. Grammdorf 1960 /WS}

$1420 \pm 40$

Coll. 1960 and subm. 1968 by K. W. Struve. Comment (K.W.S.): charcoal from cross section of rampart near Grammdorf $\left(54^{\circ} 16^{\prime} \mathrm{N}\right.$ Lat, $10^{\circ} 49^{\prime} \mathrm{E}$ Long), Germany. Intended to date early Slavonian ceramics (Struve, 1961, 1969).

\section{KI-263. Scharstorf/Ws 2, 1959}

$1515 \pm 65$

Coll. 1959, subm. 1968 by K. W. Struve. Comment (K.W.S.): part of lowest layer of wooden base of rampart, found near Scharstorf $\left(54^{\circ} 14^{\prime} \mathrm{N}\right.$ Lat, $10^{\circ} 19^{\prime} \mathrm{E}$ Long), Schleswig-Holstein, Germany. Intended to date Slavonian castles (Struve, 1961).

\section{KI-264. Warder 1959-Rost}

$1130 \pm 55$

Coll. 1959, subm. 1968 by K. W. Struve. Comment (K.W.S.): charcoal beneath duck-boards of Slavonian settlement near Warder $\left(53^{\circ} 59^{\prime}\right.$ N Lat, $10^{\circ} 23^{\prime}$ E Long), Schleswig-Holstein, Germany (Struve, 1961).

KI-87.

$2810 \pm 80$

860 B.C.

\section{KI-272.}

$2400 \pm 40$

450 B.c.

Charcoal from fireplace (r 3539 250, h 6026 250) near Fockbek/ Rendsburg, Schleswig-Holstein, Germany. Coll. and subm. 1966 (KI-87) and 1968 (KI-272) by W. Heinrich, Kiel. Fireplace was thought to be from Mesolithic period because of presence of many fragments of flint. $\mathrm{C}^{14}$ dates KI-87 and 2nd sample, KI-272, coll. 11/2 yr later did not confirm expectation.

Date list:

REFERENCES

Kiel III Willkomm and Erlenkeuser, 1968

Hänsel, K.-G., 1968, Eine C ${ }^{14}$-Messapparatur mit einem Oeschgerzählrohr zur Altersbestimmung organischer Substanzen: Diplomarbeit, Kiel.

Struve, K. W., 1961, Die slawischen Burgen in Wagrien: Offa, v. 17/18, p. 65

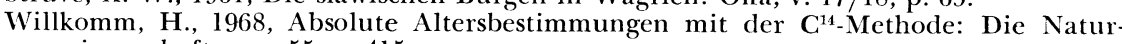
wissenschaften, v. 55, p. 415.

Willkomm, H. and Erlenkeuser, H., 1968, University of Kiel radiocarbon measurements III: Radiocarbon, v. 10, p. 328-332.

Winkelmann, W., 1959, Eine ausgedehnte jungpaläolithische Freilandsiedlung der Federmessergruppe bei Westerkappeln-Westerbeck, $\mathrm{Kr}$. Tecklenburg: Germania, v. 37 , p. 254 . 